\title{
Menopausia y síndrome metabólico
}

\section{The menopause and metabolic syndrome $X$}

\author{
Alejandro Pinzon Tovar', Carlos Celemin²
}

\begin{abstract}
Resumen
El incremento en el contenido de grasa visceral abdominal y el aumento en la resistencia a insulina de la mujer post menopáusica son eventos principales en el desarrollo del síndrome metabólico; que se define como un grupo de alteraciones clínicas que incluye la obesidad, trastornos hiperglicemicos, hipertensión y dislipidemia ${ }^{1}$. Todos estos trastornos aumentan el riesgo de enfermedad cardiovascular, siendo una de las principales causas de morbi-mortalidad en las mujeres mayores de 55 años en los países occidentales. Los estrógenos endógenos parecen ser cardio protectores y la deficiencia estrogénica de la menopausia se asocia a un sin número de alteraciones metabólicas que incrementan el riesgo cardiovascular.

En esta revisión se abordan los principales tópicos relacionados con el síndrome metabólico así como su tratamiento durante la menopausia y modificación del estilo de vida
\end{abstract}

Palabras clave: Síndrome Metabólico, Menopausia, Obesidad.

\begin{abstract}
The rise in abdominal visceral fat and the increase in resistance to insulin in postmenopausal women are main factors in the development of metabolic syndrome X, defined as a group of clinical changes which include obesity, hyperglycaemic disorders, hypertension and dyslipidaemia. All of these disorders increase the risk of cardiovascular disease, one of the main causes of morbidity and mortality in women over the age of 55 in Western countries. Endogenous oestrogen seems to be a cardioprotector and the oestrogen deficiency that occurs during the menopause is associated with a number of metabolic changes which increase the cardiovascular risk.

This work addresses the main topics related to metabolic syndrome $X$ as well as its treatment and the impact it has on lifestyle during the menopause.
\end{abstract}

Key words: Metabolic Syndrome X, Menopause, Obesity.

\section{Introducción}

La incidencia del síndrome metabólico aumenta con la edad, afecta el $40 \%$ de personas mayores de 60 años, periodo que en mujeres corresponde a la menopausia y se explica por los cambios hormonales ocurridos secundarios al hipoestrogenismo. En Colombia, se estima una población de 915.085 mujeres para el año 2015 y 1.129 .812 para el 2020 en las edades de 60 a 64 años. A nivel poblacional las principales causas de muerte son las cardiovasculares, seguidas de enfermedades neoplásicas, enfermedad cerebrovascular y diabetes. En términos generales son los hombres los más afectados ${ }^{2}$. En mujeres, el síndrome metabólico se incrementa con la menopausia y explica parcialmente la aceleración e incidencia aumentada de enfermedad cardiovascular en esta etapa de la vida femenina. Esta afirmación esta soportada con estudios realizados en diferentes países alrededor del mundo ${ }^{3,4}$. El síndrome metabólico se ha convertido en un problema de salud

1 MD. Internista, Universidad Surcolombiana Neiva. Endocrinólogo Universidad Militar Nueva Granada. Médico Internista y Endocrinólogo del Hospital Universitario Hernando Moncaleano Perdomo Neiva. Médico Internista y Endocrinólogo de ENDHO Colombia Neiva. Miembro numero ACE. Presidente capitulo eje cafetero (2014)

Correspondencia: Alejandro Pinzón Tovar. Correo electrónico: alepyto@yahoo.com

2 MD. General, Universidad del Tolima. Medico general Clínica Uros Neiva. Medico general de ENDHO Colombia Neiva.

Recibido: 08/10/2013 Revisado: 31/03/2014 Aceptado: 25/06/2015 
pública principalmente por causa de la obesidad ${ }^{5}$. En 2008 , en el Hospital Militar de Colombia, el 25\% de las pacientes con menopausia reunían criterios de síndrome metabólico; sin embargo, solo en la mitad se realizó el diagnóstico. Vale la pena mencionar que existe un subregistro del perímetro abdominal en las historias clínicas.

\section{Definiciones}

Se define la menopausia como el periodo de transición entre la edad reproductiva y la no reproductiva de la mujer, y se caracteriza por la ausencia de menstruación en 12 meses consecutivos, reflejando la pérdida de la ovulación y la supresión de la producción de estrógenos ${ }^{6,7}$. La historia menstrual es el mejor indicador del estado menopáusico.

1. Como objetivo de contar con una terminología universal, así como un sistema de estadificación para categorizar la edad ovárica, incluyendo criterios menstruales y hormonales, el Stages of Reproductive Againg Workshop (STRAW) actualización 2012, los institutos nacionales de salud, en conjunto con la Sociedad Norteamericana de Menopausia; crean un sistema que divide el proceso continuo del envejecimiento por etapas, teniendo como base el hecho de que no todas las mujeres sanas seguirán el mismo proceso, ya que podrán oscilar o saltar alguna o algunas etapas ${ }^{8}$. Última etapa reproductiva (Etapa -3 ): marca el tiempo donde la fecundidad empieza a decaer y durante el cual la mujer nota cambios en su ciclo menstrual.

2. Transición temprana a la menopausia (Etapa -2): es marcada por un incremento en la variabilidad en la duración de los ciclos menstruales, definido como una dife- rencia persistente (dentro de 10 ciclos) de 7 días o más en la duración de ciclos menstruales consecutivos.

3. Transición final de la menopausia (Etapa -1): es la ocurrencia de amenorrea por 60 días o más. Los ciclos menstruales son caracterizados por un incremento en la duración, fluctuaciones extremas en niveles hormonales y un incremento en la prevalencia de anovulación.

4. Postmenopausia temprana (Etapa +1$)$ : marca el final de un periodo de 12 meses de amenorrea, se subdivide en $+1^{\mathrm{a}},+1 \mathrm{~b}$ y $+1 \mathrm{c}$, los cuales abarcan un periodo de aproximadamente 5 a 8 años, con síntomas vasomotores y cambios hormonales (niveles elevados de FSH (Hormona Folículo Estimulante) y bajos niveles de estradiol) como representantes de este periodo.

5. Postmenopausia tardía $($ Etapa +2$)$ : representa un periodo en la cual los cambios en la función reproductiva endocrina están limitados y los procesos de envejecimiento somático son de suma importancia. Los síntomas más representativos de este periodo son sequedad vaginal y atrofia urogenital.

El síndrome metabólico fue descrito por primera vez en 1988 por Gerald Reaven, quien inicialmente lo denominó Síndrome $\mathrm{X}^{9}$. Representa una interrelación de desórdenes clínicos comunes: obesidad, resistencia a la insulina, intolerancia a la glucosa, hipertensión arterial y dislipidemia. El incremento y la redistribución de la grasa corporal durante la menopausia predisponen a las mujeres a presentar enfermedad cardiovascular y síndrome metabólico ${ }^{5,10}$. La O.M.S propone sus criterios diagnósticos para el síndrome metabólico, (Tabla 1), y el consenso Colombiano de síndrome metabólico adopta la definición de la Federación Internacional de Diabetes

Tabla 1. Criterios propuestos por la OMS para el diagnóstico del síndrome metabólico.

Parámetros principales Definición

Intolerancia a la glucosa o Diabetes Mellitus tipo?

Resistencia a la insulina con tolerancia a la glucosa normal
Glicemia de ayuno $>110 \mathrm{mg} / \mathrm{dl}$ y/o $2 \mathrm{hr}$ Post-carga $=140 \mathrm{mg} / \mathrm{dl}$

Captación de glucosa por debajo del percentil 25 en clamp euglicémico-hiperinsulinémico
Otros parámetros

Hipertensión arterial

Triglicéridos

Colesterol do HDL (CHDL)

Obesidad abdominal

Microalbuminuria

$$
\begin{aligned}
& =140 / 90 \mathrm{~mm} \mathrm{Hg} \\
& =150 \mathrm{mg} / \mathrm{dl} \\
& \text { Hombres }<35 \mathrm{mg} / \mathrm{dl} \\
& \text { Mujeres }<39 \mathrm{mg} / \mathrm{dl}
\end{aligned}
$$

Circunferencia abdominal (cresta ilíaca)

Hombres > $102 \mathrm{~cm}$; Mujeres > $88 \mathrm{~cm}$ a bien Indice de Masa Corporal (IMC) $>30 \mathrm{~kg} / \mathrm{m} 2$

Excreción urinaria de albúmina $=20 \mathrm{pg} / \mathrm{min}$ 
que incluye las mismas variables del ATP III y que considera la obesidad central como un componente esencial que debe ser valorado según la etnia (Tabla 2). Según la clasificación del ATP III se debe cumplir con tres o más de las características descriticas ${ }^{1,11}$.

La transición temprana a la menopausia está relacionada con el incremento en la acumulación de grasa corporal central (grasa visceral intra abdominal), cambio hacia un perfil lipídico más aterogénico con aumento en los niveles de lipoproteínas de baja densidad (LDL) y triglicéridos; y disminución en los niveles de lipoproteínas de alta densidad (HDL), e incremento en los niveles de insulina y glucosa. La emergencia del riesgo puede ser el resultado directo de la falla ovárica o alternativamente una causa indirecta de las consecuencias metabólicas de la redistribución de grasa central unida a la deficiencia de estrógenos ${ }^{3}$.

El índice de masa corporal incrementa con la edad y el pico ocurre entre los 50 y 59 años. En la mujer menopáusica influyen otros factores además de los cambios hormonales que contribuyen a la ganancia de peso; el aumento en la ingesta energética y la disminución de la tasa metabólica afectan la composición corporal y promueven una tendencia hacia la ganancia de peso ${ }^{12}$.

\section{Evento cardiovascular, sindrome metabolico y menopáusia}

La enfermedad cardiovascular es la principal causa de muerte en mujeres de países occidentales ${ }^{13}$. Sin embargo, la ocurrencia de la enfermedad ateroesclerótica es distinta en hombres y mujeres. La mujer pre menopáusica parece estar protegida para eventos cardiovasculares comparadas con hombres de su misma edad. Aunque la mujer menor de 50 años rara vez desarrolla enfermedad cardiovascular la incidencia en las mayores de 70 años se iguala a la de los hombres; lo anterior sugiere que la deficiencia de estrógenos causa una rápida aceleración en el incremento del riesgo ${ }^{14}$. Existe controversia sobre si la menopausia incrementa el riesgo cardiovascular independiente del

Tabla 2. Criterios para el diagnóstico clínico del síndrome metabólico.

\begin{tabular}{lll}
\hline Criterios diagnóstico & NEP ATPIII actualizado (2005) & IDF \\
\hline Requisito & 3 de los 5 factores de riesgos siguientes & $\begin{array}{l}\text { Presencia de obesidad central dada por } \\
\text { CC según pertenencia étnica más } 2 \text { de } \\
\text { los } 4 \text { factores de riesgo restantes }\end{array}$ \\
\hline Circunferencia de cintura (cm) & $\begin{array}{l}\text { CC } \\
\text { Hombres: }>40 \\
\text { Mujeres: }>88\end{array}$ & $\begin{array}{l}\text { CC (población especifica): } \\
\text { Personas de origen centro o ser americano: } \\
\text { Hombres }=90 \\
\text { Mujeres }=80\end{array}$ \\
\hline
\end{tabular}

Triglicéridos $(\mathrm{mg} / \mathrm{dl})$

TG $=150$ o en tratamiento Farmacológico

para HTG.

\begin{tabular}{|c|c|}
\hline \multirow[t]{5}{*}{ Colesterol HDL (mg/dl) } & cHDL: \\
\hline & Hombres $<40$ \\
\hline & Mujeres < 50 \\
\hline & O en tratamiento farmacológico para glicemia \\
\hline & elevada. \\
\hline Presión arterial $(\mathrm{mmHg})$ & $\begin{aligned}= & 130 / 0=85 \text { o tratamiento farmacológico } \\
& \text { para hipertensión. } \\
= & 100 \text { (incluidos sujetos con DM2) o tratamiento } \\
& \text { farmacológico para glicemia elevada. }\end{aligned}$ \\
\hline
\end{tabular}

NCEP ATP III: National Cholesterol Education Program Adult Panel Treatment. IDF: International Diabetes Federation.

DM2: diabetes mellitus tipo 2, CC: circunferencia de cintura, TG: triglicéridos, cHDL: colesterol HDL, HTG: hipertrigliceridemia. 
envejecimiento normal; algunos estudios demostraron el aumento del riesgo después de este período; por ejemplo, los investigadores de Framingham hallaron un incremento 4 veces mayor de enfermedad cardiovascular en los 10 años posteriores al inicio de la menopausia ${ }^{3}$.

El riesgo cardiovascular atribuible al síndrome metabólico parece ser especialmente alto en mujeres y se ha estimado que la mitad de todos los eventos cardiovasculares están relacionados con la presencia de síndrome metabólico ${ }^{5}$. El síndrome metabólico no es una simple entidad, más bien, es una constelación de factores de riesgo relacionados. Las fisiopatología del síndrome metabólico incluye acumulación de la grasa abdominal visceral, resistencia a la insulina, hipertensión y dislipidemia; todas relacionadas con enfermedad cardiovascular ${ }^{2,5,15}$. Se estima que el síndrome metabólico afecta aproximadamente del 20 al 30\% de la población de edad media y su prevalencia esta en incremento gracias al aumento de la obesidad y al estilo de vida sedentario ${ }^{16}$. El estado menopáusico se asocia con un aumento del $60 \%$ en la incidencia de síndrome metabólico ${ }^{3}$.

En la menopausia, los cambios metabólicos y hormonales ocurren durante varios años y varían ampliamente entre las mujeres. Sutton-Tyrrell y colaboradores demostraron que el $45 \%$ de las mujeres menopáusicas tenían un engrosamiento significativo clínicamente de la íntima de la carótida. La calcificación aórtica, otra medida para ateroesclerosis, fue mayor en mujeres menopáusicas y las extensión de las calcificaciones incrementaban con los años post menopáusicos. De igual forma, los depósitos de calcio en las arterias coronarias medidos por tomografía computada, fueron la mitad que en los hombres hasta los 60 años, luego de lo cual la diferencia disminuyo ${ }^{3}$.

La alteración en el metabolismo de los lípidos sumado al déficit de estrógenos puede determinar un papel importante en el riesgo cardiovascular de la mujer en menopausia ${ }^{13}$. Otros efectos directos de la deficiencia de estrógenos que influyen sobre el riesgo cardiovascular son el engrosamiento de la pared arterial y las alteraciones en la fibrinólisis. Todos estos factores empeoran el riesgo cardiovascular futuro ${ }^{3,4}$.

\section{Menopausia y composicion corporal.}

Se ha establecido en mujeres pre menopáusicas que la actividad física reducida y el incremento en la adiposidad son predictores independientes de muerte ${ }^{17}$. El aumento de grasa central o intra abdominal (androide o en forma de manzana) se considera un factor de riesgo para síndrome metabólico y enfermedad cardiovascular independiente de la obesidad en general. Esta distribución de grasa corporal se ha asociado con un riesgo aumentado para desarrollar diabetes, hipertrigliceridemia con acumulación de LDL pequeña y densa, hipertensión y eventos cardiovasculares. Los estrógenos promueven la acumulación de grasa glúteo femoral (ginecoide o en forma de pera) y su pérdida junto con la menopausia se relaciona a acumulación de grasa central ${ }^{3,13,18}$. El dimorfismo sexual en la distribución de grasa corporal puede explicar parcialmente el marcado riesgo cardiovascular en hombres comparado con mujeres pre menopáusicas. Se ha determinado que las mujeres con incremento en la grasa visceral (deposito de tejido graso principalmente en hígado, musculo y páncreas) aumentan las anormalidades metabólicas y su mortalidad cardiovascular ${ }^{3}$.

Esta grasa visceral implica la formación en el tejido graso de sustancias químicas llamadas adipoquinas, que favorecen estados proinflamatorios y protrombóticos, que a su vez van a contribuir al desarrollo de insulino resistencia, hiperinsulinemia, alteración en la fibrinólisis y disfunción endotelial.

Una adipoquina en particular, la adiponectina, a diferencia del resto, se encuentra disminuida; esta situación asociada a un incremento del nivel de triglicéridos, disminución de colesterol HDL, elevación de apolipoproteína B y presencia de partículas pequeñas y densa de LDL, contribuyendoye al estado aterotrombotico que representa el perfil inflamatorio de la adiposidad visceral. Las concentraciones de adiponectina están inversamente relacionadas a la obesidad y a la resistencia de la insulina ${ }^{13}$. La menopausia se asocia también con la reducción de masa corporal magra, la disminución en la capacidad para la actividad física contribuye a la reducción de la masa magra y al incremento de la adiposidad central ${ }^{3,19}$.

\section{Lipidos y menopausia}

La cantidad de grasa abdominal esta asociada con incremento en la resistencia a la insulina, el aumento en el nivel de ácidos grasos libres y la disminución en los niveles de la adiponectina (Figura 1). Estos factores elevan la secreción de partículas que contienen apolipoproteína B (apo B), produciendo hipertrigliceridemia y aumento en la actividad de la lipasa hepática con predominio de LDL pequeña y densa, y reducción en los niveles de HDL; un perfil similar al de la menopausia ${ }^{3,13}$. La mujer menopáusica eleva el colesterol total, el colesterol LDL, los triglicéridos y los niveles de lipoproteína a (Lp a); mientras que el colesterol HDL disminuye a un nivel más bajo que el hallado en mujeres premenopáusicas ${ }^{3,4,10}$. Aunque los niveles elevados de LDL no son un componente del síndrome metabólico, incrementan en un 10 a $20 \%$ con la menopáusia y el mayor cambio ocurre durante la transición de pre a menopáusia. La LDL pequeña y densa, aumenta en un 30 a $49 \%$ en el estado menopáusico, sus niveles elevados se relacionan con un incremento en el riesgo de infarto de miocardio y con la severidad de la enfermedad cardiovascular. El riesgo de eventos cardiovasculares es tres veces mayor en mujeres con LDL pequeña y densa elevada, comparado con LDL grandes y solubles.

Varios estudios han demostrado que el nivel de triglicéridos aumenta con la transición a la menopausia y esta elevación parece llevarse a cabo en el período temprano post menopáusico. Un incremento del $16 \%$ en el nivel de triglicéridos parece ser el mejor predictor de riesgo cardiovascular. El aumento en los triglicéridos durante la menopausia está relacionado con el incremento de la grasa abdominal y resistencia a la insulina. 


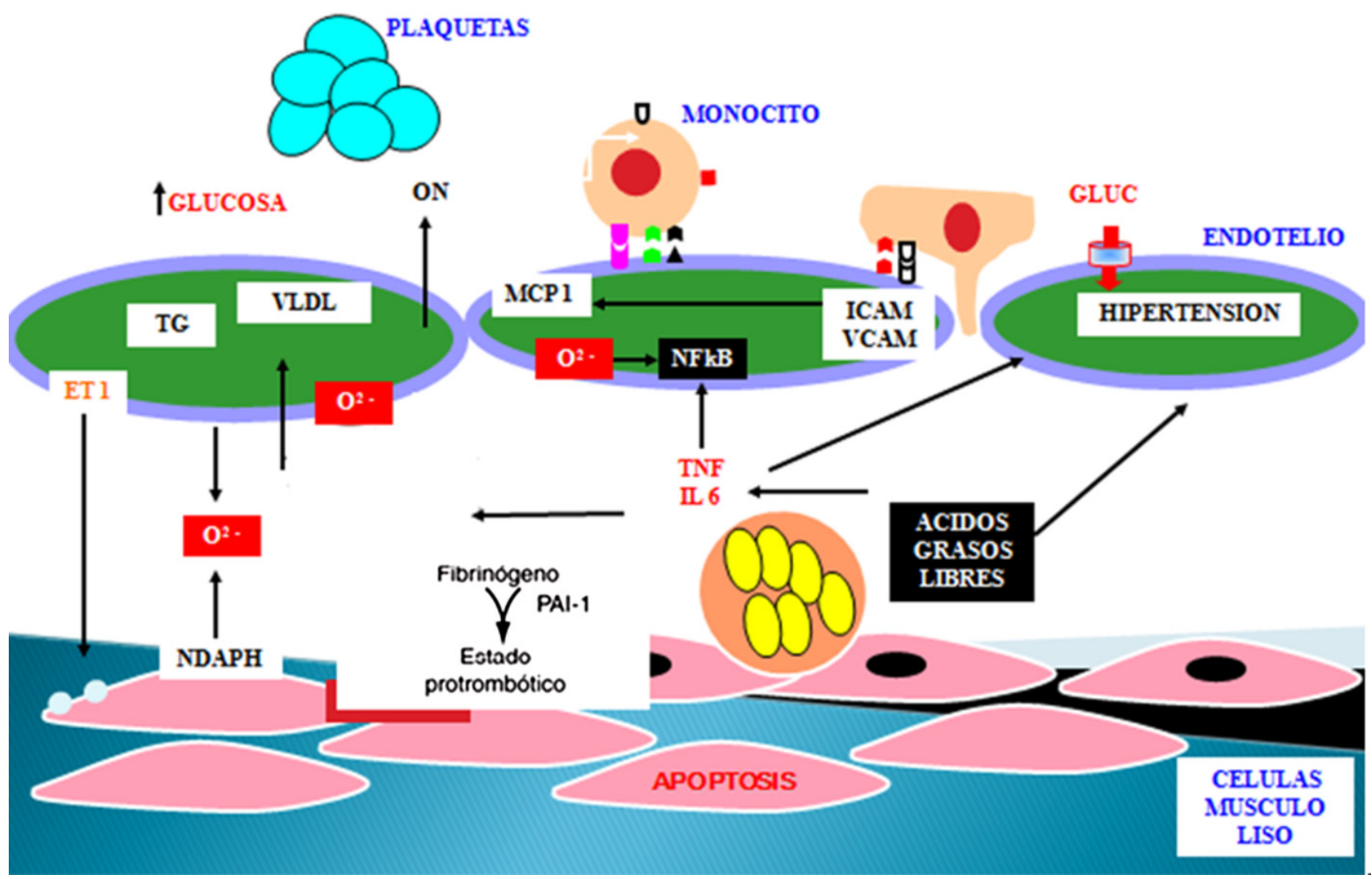

Figura 1. Fisiopatología del síndrome metabólico.

Los ácidos grasos libres (AGL) son liberados a partir de la masa total de tejido adiposo. En el hígado, la presencia de dichos ácidos aumenta la producción de glucosa, triglicéridos (TG) y se secreta lipoproteínas de muy baja densidad (VLDL). Estas anomalías incluyen una disminución en el colesterol de las lipoproteínas de alta densidad (HDL). Los AGL disminuyen la sensibilidad a la insulina, al inhibir la captación de glucosa por los músculos. Los incrementos de glucosa circulante y AGL hacen que haya un hiperinsulinemia, esta última intensifica la reabsorción de sodio y también aumenta la actividad del sistema nervioso simpático, y contribuye a la hipertensión y a las concentraciones de AGL. El estado proinflamatorio se sobreañade y contribuye a la resistencia a la insulina generado por el exceso de AGL. La secreción de interleucina 6 (IL-6) y factor de necrosis tumoral alfa (FNT á) generado por adipocitos y macrófagos derivados de monocitos intensifican la resistencia a la insulina y la lipólisis. Las citocinas y los AGL también aumentan la producción de fibrinógeno por el hígado y de la producción de inhibidor del activador de plasminógeno 1 por adipocitos, lo que origina un estado protrombótico.

Los niveles de HDL caen durante la menopausia, las HDL2, las más anti aterogénicas disminuyen en un 25\%, mientras que los niveles de HDL3 incrementan. Las partículas de HDL2 son las más grandes, solubles y cardioprotectoras de todas las especies de HDL. La relación inversa entre los niveles de colesterol HDL y la adiposidad abdominal, parece ser dependiente de los niveles cambiantes de HDL2.

La lipoproteína a $\mathrm{Lp}(\mathrm{a})$, una partícula parecida a las LDL, con una estructura homóloga al plasminógeno, no es frecuentemente medida en la práctica clínica pero se ha visto que su nivel predice eventos cardiovasculares independientes del nivel de LDL. Las concentraciones de Lp(a) son genéticamente determinados y se estima que durante la menopausia los niveles aumentan entre 25 y $50 \%$. Esta ele- vación puede reflejar el hecho que los niveles de Lp(a) están influenciados por esteroides sexuales y que retornan a niveles premenopáusicos en mujeres que reciben reemplazo de estrógenos.

El incremento en la prevalencia de la LDL pequeña y densa durante la menopausia puede ser explicado por el aumento en la actividad de la lipasa hepática. El nivel de estrógenos endógenos es inverso a la actividad de esta enzima. El aumento en la actividad de la lipasa hepática produce lipoproteínas más pequeñas y densas y por ende más aterogénicas. La lipoproteína lipasa, hidroliza triglicéridos generando ácidos grasos libres que pueden almacenarse en adipocitos o servir como sustrato energético. Esta hormona posee un incremento leve de su actividad durante la menopausia ${ }^{3,10}$. 


\section{Resistencia a la insulina durante la menopausia}

La obesidad abdominal se asocia marcadamente con la resistencia a la insulina, la hiperinsulinemia compensatoria incrementa el riesgo de desarrollar diabetes mellitus 2 independiente del contenido individual de grasa corporal total ${ }^{10}$.. El incremento en los niveles de ácidos grasos libres altera la captación en los tejidos periféricos de la glucosa, incrementa la gluconeogénesis y reduce la depuración hepática de insulina. Varios grupos han demostrado que el incremento en los niveles de insulina de ayuno y el incremento en los niveles de glucosa de ayuno en mujeres menopáusicas comparado con los niveles de mujeres premenopáusicas podrían reflejar la resistencia a la insulina empeorada durante la menopausia. Sin embargo, la sensibilidad de la insulina se deteriora a medida que avanza la edad e incrementa la obesidad central, haciendo difícil discernir el efecto de la menopausia sobre este proceso ${ }^{2}$. Aunque se sabe que la resistencia a la insulina ocurre en mujeres obesas y con síndrome metabólico, no existe un mecanismo practico para diagnosticar esta condición ya que los niveles de insulina no son de mayor utilidad. El mecanismo por el cual el aumento en la grasa abdominal contribuye a la resistencia a la insulina involucra adipocitoquinas (adiponectina, leptina, resistina) y péptidos gástricos como la ghrelina. Las adipocitoquinas son actualmente determinantes importantes de enfermedad cardiovascular, pero su relación con obesidad y/o resistencia a la insulina en mujeres menopáusicas con síndrome metabólico aún no ha sido determinada $^{5,20}$.

DeNino, hallo que la sensibilidad alterada a la insulina en la mujer no aparece hasta, una vez acumule un nivel de grasa visceral aproximado a los vistos en los hombres; al parecer debe existir un posible umbral de grasa abdominal para que se produzca resistencia a la insulina.

Chu evalúo 37 mujeres obesas menopáusicas con síndrome metabólico y encontró que el 70\% tenían niveles elevados de insulina en ayunas y $73 \%$ tenían resistencia a la insulina con valores de HOMA > 47 (HOMA= insulin [pmol/L] x glucose $[\mathrm{mmol} / \mathrm{L}] / 135)$. Al compararlas con el grupo control de mujeres obesas premenopáusicas los niveles de adiponectina y ghrelina fueron más bajos y los de leptina y resistina estaban elevados. Solo la adiponectina cambia significativamente entre las obesas premenopáusicas y las mujeres normales, y son precisamente sus valores anormales los que más se relacionan con riesgo cardiovascular en la mujer menopáusica con síndrome metabólico. Una de las limitaciones de este estudio fue no estudiar mujeres menopáusicas con peso normal para comparar los niveles de adiponectina. 5 .

\section{Menopausia, sistema fibrinolítico e inflamación}

La actividad fibrinolítica es un balance entre los activadores del plasminógeno y sus inhibidores. Una actividad elevada en el inhibidor del activador del plasminógeno tipo 1 (PAI-1) causa prolongación en el tiempo de lisis del coagulo y potencia los estados de trombosis. El PAI-1 es producido en el hígado y el tejido adiposo, particularmente en el adipocito visceral y se cree que es también un marcador de resistencia a la insulina. La mujer menopáusica tiene niveles elevados de PAI-1 indicando que la disminución en los niveles de estrógenos se asocia con una disminución en el potencial fibrinolítico. De hecho, los estudios epidemiológicos muestran de manera consistente una asociación entre el uso de estrógenos y la disminución de las enfermedades cardiovasculares en mujeres posmenopáusicas. Además los estrógenos producen un perfil favorable de lipoproteínas, favorecen la vasodilatación, inhiben la respuesta a la lesión vascular y reducen la ateroesclerosis 21 .

Sin embargo estudios prospectivos con asignación al azar han indicado de manera inesperada un incremento inicial en la incidencia de cardiopatía y apoplejía en mujeres postmenopáusicas de edad avanzada ${ }^{21}$, tratadas con estrógenos conjugados y progestágenos, aunque la tendencia se corrigió con el paso del tiempo.

En el estudio WHI (The Women's Health Initiative Study), las mujeres sin cardiopatía coronaria recibieron tratamiento con estrógenos más progestágenos, se observaron efectos protectores sólo cuando el tratamiento de sustitución hormonal inició en los 10 primeros años de iniciada la menopausia.

La proteína $\mathrm{C}$ reactiva (PCR), un marcador subclínico de la presencia e intensidad de inflamación que predice independientemente el riesgo de enfermedad cardiovascular tanto en hombres como en mujeres, se relaciona directamente con el contenido de grasa corporal total y de grasa visceral. La inter leuquina 6 es una citoquina pro inflamatoria producida por macrófagos y monocitos que induce la producción de PCR. Sus niveles incrementados están relacionados con incremento en el riesgo de muerte cardiovascular ${ }^{22-24}$.

Las mujeres que desarrollan resistencia a la insulina con incremento de LDL pequeña y densa, y aumento en los niveles de PAI-1 pueden reflejar una predisposición genética que se halla enmascarada por el efecto de los estrógenos y que se manifiesta luego de la menopausia.

\section{Tratamiento de síndrome metabólico durante la menopausia}

Las mujeres menopáusicas que cumplen criterios para diagnóstico de síndrome metabólico deben ser tratadas agresivamente para reducir el riesgo cardiovascular. El enfoque terapéutico debe combinar cambios en el estilo de vida y la implementación de terapia farmacológica ${ }^{3,25-27}$.

Con respecto a los estilos de vida saludable, los carbohidratos deben sumar una cantidad importante de las calorías totales $(55-60 \%)^{25}$, minimizando los carbohidratos simples refinados (azúcar, miel, melaza, y otros), mientras que se incrementan los carbohidratos complejos (vegetales, frutas y granos enteros). Los edulcorantes pero aquellos con bajo contendió de sodio deben ser seleccionados.

Las proteínas animales son las preferidas por su alto valor biológico $(0.8 \mathrm{a} 1 \mathrm{~g} / \mathrm{kg})$, pero las legumbres y los cereales.

Las grasas de la dieta no deben sobrepasar el 30\% de las calorías totales, con menos del 10\% de grasas saturadas, $10 \%$ polisaturadas (aceite vegetal, frutas secas y pescado) y 
monoinsaturadas mas del 10\% (aguacate, olivas, pollo, cerdo $)^{25}$.

Vitaminas elementos trazos deben ser tomados como una recomendación general, al igual que minerales deben incluir sodio $(2-3 \mathrm{~g})^{25}$.

La terapia de reemplazo hormonal fue una opción para el tratamiento de mujeres menopáusicas con síndrome metabólico ya que se mejoraban muchas de las alteraciones metabólicas. Existen datos experimentales que indican que los estrógenos poseen efectos neuroprotectores, antiescleróticos y vasodilatadores $^{28}$. Sin embargo, es claro que el tratamiento con estrógenos orales incrementa el riesgo de enfermedad tromboembólica en mujeres sanas 2 veces y este multiplica los factores de riesgo basales que incluyen la edad, índice de masa corporal aumentada, trombofilias, cirugía, e inmovilización. En el estudio WHI, por ejemplo una combinación de estrógenos con progestágenos ocasiono un aumento de 8 veces el riesgo de apoplejía y se evidencio un incremento similar en tromboembolismó pulmonar ${ }^{21}$. Cabe señalar que los estrógenos transdérmicos no incrementan el riesgo de episodio tromboembolicos.

Esta información es consistente con los datos mostrados con la terapia de remplazo hormonal, la cual incrementa la producción de proteínas de coagulación mediante la vía del efecto de primer paso hepático, aquella que no es replicada con la terapia transdermica ${ }^{29}$.

El engrosamiento de la pared carótidea es indicador de ateroesclerosis de la carótida, su aumento después de la menopáusia podría determinar la importancia del nivel estrogénico. A pesar de que los estrógenos aumentan el flujo sanguíneo cerebral y reducen la resistencia vascular, es incierto el papel protector de la terapia de reemplazo hormonal sobre los eventos cardiovasculares 21,30 . El concepto de la terapia de remplazo hormonal puede reducir el riesgo cardiovascular fue basado en parte en grandes estudios observacionales, estos demostraron una reducción clínicamente del $35 \%$ en mujeres postmenopáusicas en quienes tomaban terapia de remplazo hormonal. Los datos del WHI (Women's Health Initiative) sobre el aumento del riesgo cardiovascular en usuarias de terapia de reemplazo con estrógeno progestágenos, no recomiendan esta terapia como medida preventiva ${ }^{3}$.

\section{Modificación en el estilo de vida}

La pérdida de peso y la actividad física son la piedra angular de la terapia. Aún pérdidas modestas de peso muestran mejoría en la resistencia a la insulina y en la adiposidad visceral abdominal $^{25-27}$. Idealmente la pérdida de grasa abdominal se alcanza con ejercicio aeróbico (Eje: caminar) y su realización regular mejora la sensibilidad a la insulina independiente de la pérdida de peso total. Aunque existe evidencia limitada, el objetivo de la terapia es promover una actividad periódica, regular y prolongada de baja intensidad para mantener el peso y reducir el tejido adiposo visceral más que para lograr una meta de pérdida de peso global ${ }^{3,31}$. Disminuir el nivel de LDL sigue siendo el objetivo primario ${ }^{32}$, pero reducir el nivel de triglicéridos es importante para reducir el riesgo cardiovascular. El ácido nicotínico y los fibratos actúan reduciendo los niveles de triglicéridos y aumentando los niveles de HDL; el primero puede elevar la glucosa en algunos pacientes. La terapia combinada con estatinas puede incrementar el riesgo de presentar efectos adversos.

Es importante recordar que las anormalidades lipídicas relacionadas al síndrome metabólico pueden ser sutiles, y el interés actual en establecer el tamaño de las partículas de LDL puede identificar las mujeres post menopausicas con mayor riesgo cardiovascular para establecer una terapia hipolipemiante más agresiva.

\section{Conclusiones}

Actualmente se sabe que múltiples factores de riesgo cardiovascular pueden surgir durante la menopausia, sin embargo, los rasgos característicos del síndrome metabólico pueden estar presentes aún antes. La resistencia a la insulina es una característica prominente del síndrome metabólico en mujeres menopáusicas, sin embargo, su diagnóstico con métodos clínicamente útiles se hace difícil. La hiperglucemia en pacientes con o sin el diagnóstico de Diabetes Mellitus está asociada a alta morbilidad y mortalidad. ${ }^{33}$ El aumento en el IMC puede reflejar las anormalidades en las adipocitoquinas, los niveles elevados de triglicéridos y bajos de HDL; que incrementan el riesgo cardiovascular.

El aumento en la prevalencia del síndrome metabólico a nivel mundial es alarmante, con una importante taza de morbi-mortalidad demostrada por incremento de la enfermedad ateroesclerótica subclínica aun sin el diagnostico de diabetes. Requiriendo de un manejo multidisciplinario, donde la piedra angular sea los estilos de vida saludable ${ }^{25-27}$, que implique el control del peso como objetivo principal, como método barato y efectivo en mujeres posmenopáusicas dado sus cambios conformacionales y disposición de tejido adiposo; aunado a los cambios proinflamatorios y protrombóticos que llevan a aumento en la resistencia a la insulina, cambios endoteliales y aumento en el riesgo cardiovascular.

\section{Referencias}

1. Expert panel on detection evaluation, and treatment of high blood cholesterol in adults: Executive summary of the third report of the National Cholesterol Education Program (NCEP) expert panel on detection, evaluation, and treatment of high blood cholesterol in adults (Adult Treatment Panel III). JAMA 2001;285:2558.

2. Liberopoulos, E. N. Diagnosis and management of the metabolic syndrome in obesity. Obesity reviews 6, 2005; 283-296.

3. Carr, M. C. The Emergence of the Metabolic Syndrome with Menopause. J Clin Endocrinol Metab 2003; 88: 2404-2411.

4. Kim, H. M. The Effect of Menopause on the Metabolic Syndrome Among Korean Women. Diabetes Care 2007; 30: 701-706. 
5. Chu, M. C. Insulin resistance in postmenopausal women with metabolic syndrome and the measurements of adiponectin, leptin, resistin, and ghrelin. American Journal of Obstetrics and Gynecology. 2006; 194: 100-4.

6. Bulun, S. E. The physiology and pathology of the female reproductive axis. In: Larsen, P. Williams textbook of. Endocrinology. Tenth edition. Cap. 16. 2003; 587-664.

7. WU, J. M. Ovarian Aging and Menopause: Current Theories, Hypotheses, and Research Models. Exp Biol Med 2005; 203: 818-828.

8. Harlow SD, Gass M, Hall JE, Lobo R, Maki P, Rebar RW, et al, for the STRAW+10 Collaborative Group. Executive summary of the stages of reproductive aging workshop +10: addressing the unfinished agenda of staging reproductive aging. Menopause: The Journal of The North American Menopause Society 2012; 97(4):1159-68

9. López, J. C. Síndrome metabólico e infertilidad. En: Pérez, L. E. Infertilidad y endocrinología reproductiva. 3ra Edición. Basada en evidencias. Cap. 18. 2007. 267-278.

10. Mauriége, P. Subcutaneous Adipose Tissue Metabolism at Menopause: Importance of Body Fatness and Regional Fat Distribution. J Clin Endocrinol Metab 2000; 85: 2446-2454.

11. Ardila, E. Consenso Colombiano de Síndrome Metabólico. Asociación Colombiana de Endocrinología. 2006.

12. POSITION STATEMENT. Estrogen and progestogen use in postmenopausal women: July 2008 position statement of The North American Menopause Society. Menopause: The Journal of the North American Menopause Society. Vol. 15, No. 4, 2008; pp. 584/602.

13. Tamakoshi. K. The transition to menopause reinforces adiponectin production and its contribution to improvement of insulin-resistant state. Clinical Endocrinology. 2007;66:65-71.

14. Dubey, R. K. Vascular consequences of menopause and hormone therapy: Importance of timing of treatment and type of estrogen. Cardiovascular Research. 2005;66: 295-306.

15. Coylewright, M. Menopause and Hypertension: An AgeOld Debate. Hypertension. 2008; 51:952-959.

16. Kopelman, P. Health risks associated with overweight and obesity. Obesity reviews 8 (Suppl. 1) 2007;13-17.

17. Hu, F. B. Adiposity as Compared with Physical Activity in Predicting Mortality among Women. N Engl J Med. 2004; 351:2694-703.

18. Cho, J. Postmenopausal status according to years since menopause as an independent risk factor for the metabolic syndrome. Menopause. 2008; 15(3): 524-529.

19. Sowers, M. Changes in Body Composition in Women over Six Years at Midlife: Ovarian and Chronological Aging. J Clin Endocrinol Metab 2007;92:895-901.

20. Hadii, P. The influence of menopause and body mass index on serum leptin concentrations. European Journal of Endocrinology. 2000;143:55-60.
21. Santen RJ et al. 2010, Postmenopausal Hormone Therapy: An Endocrine Society Scientific Statement. The Journal of Clinical Endocrinology \& Metabolism 95, Supplement 1:S1-S66

22. Hoene, M. The role of interleukin- 6 in insulin resistance, body fat distribution and energy balance. Obesity reviews 9, 2008; 20-29.

23. Rachon, D. Effects of oestrogen deprivation on interleukin6 production by peripheral blood mononuclear cells of postmenopausal women. Journal of Endocrinology, 2002; 172:387-395.

24. Pfeilschifter, J. Changes in Proinflammatory Cytokine Activity after Menopause. Endocrine Reviews 2002; 23 : 90-119.

25. López Jaramillo et al. Latin American Consensus on Hypertension in patients with Diabetes type 2 and Metabolic Syndrome. Journal of Hypertension 2013; 31:223-238

26. James PA, Oprail S, Carter BL, Cushman WC, DennisonHimmelfarb C, Handler J et al. 2014 Evidence-Based Guideline for the Management of High Blood Pressure in Adult: Report From the Panel Members Appointed to the Eighth Joint National Committee (JNC 8). JAMA. $2014 ; 311(5): 507-520$.

27. American Diabetes Association. Standards of medical care in diabetes, 2015. Diabetes Care. 2015;38(Suppl 1): S1-S2.

28. Majmudar, N. G. Effects of the Menopause, Gender, and Estrogen Replacement Therapy on Vascular Nitric Oxide Activity. J Clin Endocrinol Metab 2000; 85: 1577-1583.

29. Teede HJ, McGrath BP, Smolich JJ, Malan E, Kotsopoulos D, Liang YL, Peverill. RE 2000 Postmenopausal hormone replacement therapy increases coagulation activity and fibrinolysis. Arterioscler Tromb Vasc Biol 20:1404-1409.

30. Pines, A. Menopause and ischaemic stroke: basic, clinical and epidemiological considerations. The role of hormone replacement. Human reproduction update, $2002 ; 8(2)$ : $161-168$.

31. Brown, T. Prevention of obesity: a review of interventions. Obesity reviews 8 (Suppl. 1), 2007; 127-130.

32. Stone NJ, Robinson J, Lichtenstein AH, Bairey Merz CN, Lloyd-Jones DM, Blum CB, McBride P, Eckel RH, Schwartz JS, Goldberg AC, Shero ST, Gordon D, Smith Jr SC, Levy D, Watson K, Wilson PWF, 2013 ACC/AHA Guideline on the Treatment of Blood Cholesterol to Reduce Atherosclerotic Cardiovascular Risk in Adults, Journal of the American College of Cardiology (2013), doi: 10.1016/i.jacc.2013.11.002.

33. Pinzón, A. Bases para realizar un protocolo de control glucémico. RFS, vol 4. N. 2. Julio-Diciembre 2012. 